\title{
Optimization of Tremblay's Battery Model Parameters for Plug-in Hybrid Electric Vehicle Applications
}

\author{
Y. Zhang, Student Member, IEEE \\ S. Lyden, Member, IEEE \\ B.A. León de la Barra, Senior Member, IEEE \\ School of Engineering and ICT \\ University of Tasmania \\ Hobart, Australia \\ yuchuanz@utas.edu.au
}

\author{
M. E. Haque, Senior Member, IEEE \\ School of Engineering \\ Deakin University, Waurn Ponds Campus \\ Geelong, Australia \\ enamul.haque@deakin.edu.au
}

\begin{abstract}
Accurate modeling of batteries for plug-in hybrid vehicle applications is of fundamental importance to optimize the operation strategy, extend battery life and improve vehicle performance. Tremblay's battery model has been specifically designed and validated for electric vehicle applications. Tremblay's parameter identification method is based on evaluating the three remarkable points manually picked from a manufacturer's discharge curve. This method is error prone and the resultant discharge curve may deviate significantly from the experimental curve as reported in previous studies. This paper proposes to use a novel quantum-behaved particle swarm optimization (QPSO) parameter estimation technique to estimate the model parameters. The performance of QPSO is compared to that of genetic algorithm (GA) and particle swarm optimization (PSO) approaches. The QPSO technique needs less tuning effort than other techniques since it only uses one tuning parameter. Reducing the number of iterations should be a welcome development in most applications areas. Results show that the QPSO parameter estimation technique converges to acceptable solutions with fewer iterations than that obtained by the GA and the PSO approaches.
\end{abstract}

Index Terms-Tremblay's battery model, parameter identification, genetic algorithm, particle swarm optimization, quantum-behaved particle swarm optimization.

\section{INTRODUCTION}

With increasing fossil fuel prices and concerns about climate change [1]-[3], automobile manufacturers have equipped vehicles with diversified energy sources [4], [5]. Plug-in hybrid electric vehicles (PHEVs) have been introduced to the market to facilitate electrification of transportation [6], [7]. PHEVs have significant advantages in flattening the net demand profile [8] and reducing carbon emissions [9]. The PHEV batteries can be plugged into the domestic electricity grid as well as being charged from renewable energy sources [2]. PHEV batteries act as energy storage to counter the intermittency of renewable energy sources as well as to supply power back to the grid via vehicle-to-grid (V2G) technologies. A battery is one of the key elements of a PHEV used to provide electrical energy to the motor to propel the vehicle. A PHEV battery's steady-state and dynamic performance might be accurately predicted by a suitable battery model which is critical for simulation studies. The characteristics of a battery can be analyzed and the battery state-of-charge (SOC) prediction can be performed in different driving scenarios through simulation studies.

Modeling of PHEV batteries is a research area that has been studied extensively throughout the literature. Some earlier examples in this area have been demonstrated in [10]-[13]. A small subset of more recent research is described in [8], [14], [15]. These PHEV battery models vary widely in terms of complexity and accuracy [15].

In [16], modeling strategies for batteries are summarized into three categories: experimental, electrochemical and electric circuit-based models [8], [16]. Experimental and electrochemical models cannot accurately represent the dynamics of vehicle batteries in terms of the SOC estimations of battery packs according to [16]. Electrochemical models emulate the static characteristics of a PHEV battery using mathematical equations that relate to the chemical reactions inside the battery [17]. These models cannot accurately simulate the battery's dynamic response [18] and require high computational power to solve the associated nonlinear partial differential equations [19]. The circuit-based models provide reasonable accuracy and robustness in simulating the dynamics of the battery [20]-[22]. Their model parameters can be estimated based on experimental data. A dedicated automated test system is used to acquire the data in [23], these data include the responses of a battery at different discharge current levels and SOC conditions [23]. Tremblay's model is a widely used battery model in research on battery energy storage systems [8], on-board electric vehicle batteries [15], [19] and wireless sensor networks [17]. The model incorporates both empirical and electrochemical relationships between SOC and battery terminal voltage. It is embedded into Matlab/Simulink as a standard library block with predefined battery model parameters [16], [24]. It has also been validated for electric vehicle applications using experimental data [15]. This paper proposes to use the quantum-behaved particle swarm 
optimization (QPSO) parameter estimation technique to provide a more accurate estimation of Tremblay's battery model parameters. To the authors' best knowledge, this approach has not yet been applied in the context of Tremblay's model parameterization. The results obtained using the QPSO approach are compared against those obtained using the genetic algorithm (GA) [13] and particle swarm optimisation (PSO) [15] approaches. The model parameters and the root weighted residual sum of squares (RWRSS) between the model curves and the discharge curves obtained from static capacity tests conducted by Idaho National Laboratory [25] are used to contrast the different sets of results.

The rest of the paper is organized as follows. Section II will explain Tremblay's model that emulates a typical battery discharge characteristic and the method described in [16], [24] to identify its model parameters and other parameter optimization techniques [8], [13], [15], [26]. Section III provides a brief review of GA, PSO, and QPSO parameter estimation algorithms and provides detailed procedures for identifying the model parameters. Section IV compares and analyses the results among the three parameter estimation techniques. Finally, Section $\mathrm{V}$ discusses the results and presents a summary and possible future work.

\section{PHEV BATTERY MODEL}

\section{A. Tremblay's Model}

Tremblay's model expresses the battery voltage during discharging by [16], [24]:

$$
V_{\text {dis }}=E_{o}-K \frac{Q}{Q-i t} \cdot i t-R \cdot i+A \exp (-B \cdot i t)-K \frac{Q}{Q-i t} \cdot i^{*}
$$

where $E_{o}$ is the battery constant voltage; $K$ is the polarization constant $(V / A h)$ or polarization resistance $(\Omega) ; Q$ is the maximum battery capacity $(A h)$; it $=\int i d t$ is the actual battery charge $(A h)$; the parameter $A$ is the exponential zone amplitude $(V) ; i^{*}$ is the filtered current $(A) ; B$ is the exponential zone time constant inverse $(A h)^{-1} ; R$ is the internal resistance $(\Omega) ; i$ and $V_{\text {dis }}$ are the battery current and voltage during discharge, respectively. The current is positive for a discharging process whereas the current is negative during a charging process. The discharge voltage of the battery is decomposed into five components, which are the battery constant voltage $E_{o}$, the voltage drop on the internal resistance $R \cdot i$, the polarization voltage affected by battery charge $K \frac{Q}{Q-i t} \cdot i t$, the polarization voltage affected by filtered current $K \frac{Q}{Q-i t} \cdot i^{*}$, and the exponential zone voltage $A \exp (-B$. it). The filtered current $i^{*}$ can be expressed as the first order step response of the battery current $i$, i.e., $i^{*}=i \cdot\left(1-e^{-\frac{t}{\tau}}\right)$, where $\tau$ is the battery response time. Fig. 1 shows a typical PHEV battery nominal discharge characteristic generated by Tremblay's model, where the remarkable points are clearly shown.

\section{B. Model Parameters Identification}

Tremblay proposed a parameter estimation method described in [16], [24] to estimate the model parameters. This method utilizes the three remarkable points (shown in Fig. 1),

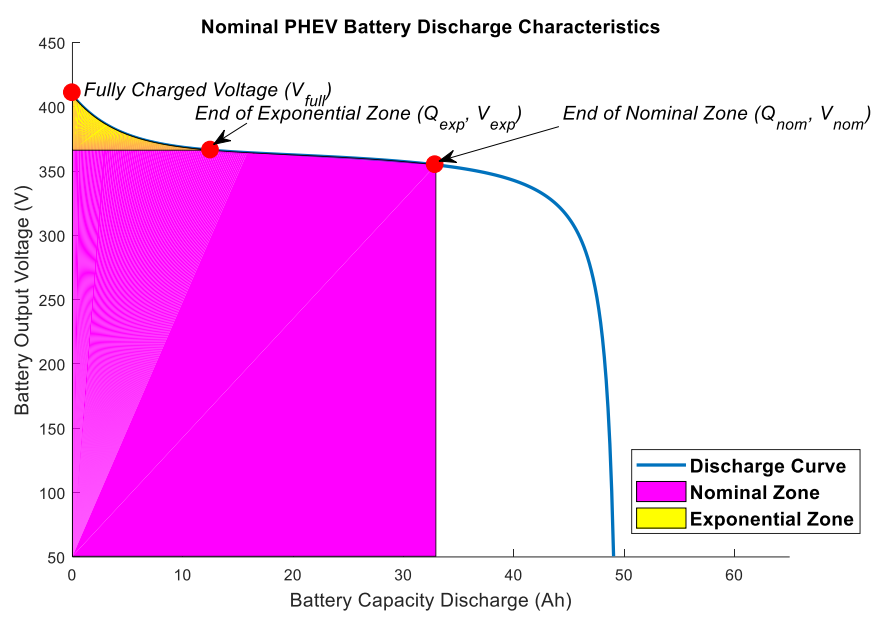

Fig. 1. A typical battery discharge characteristic.

namely the fully charged voltage $\left(V_{f u l l}\right)$, end of exponential zone $\left(Q_{\text {exp }}, V_{\text {exp }}\right)$ and end of nominal zone $\left(Q_{\text {nom }}, V_{\text {nom }}\right)$ that are manually sampled from the typical discharge characteristic on the manufacturer's datasheets. The parameters $A, B, K$, and $E_{o}$ in (1) are determined from the three remarkable points using several approximation equations. Thus, this approach is quite subjective and error-prone in terms of locating the positions of the remarkable points [15]. The remarkable points on the extracted discharge curve may not be easily identifiable and are highly susceptible to human errors. Specifically, the parameters $Q_{\text {nom }}, V_{\text {exp }}$ and $Q_{\text {exp }}$ are difficult to determine from the manufacturer's discharge curve by visual analysis as indicated in [27]. The discharge characteristic obtained from the manufacturer's datasheets may not reflect the actual discharge curve of the battery since model parameters are dependent on battery life and operational environment [8]. Thus, parameter identification for PHEV batteries needs to be conducted over time and corresponding adjustments of model parameters must be made to account for changes attributed to battery aging [15].

A variety of parameter estimation techniques are proposed in the literature for the parameterization of battery models. A particle swarm optimization (PSO) technique is presented in [15] to estimate the model parameters of Tremblay's model. A hybrid optimization technique is proposed in [8] that utilises a stochastic and a deterministic algorithm to identify the parameters of Tremblay's model. A GA approach is used in [13] to identify the parameters of an improved Thévenin battery model to account for the effects of electrochemical polarization and concentration polarization. A simulated annealing (SA) algorithm approach is proposed in [27] for Tremblay's model parameterization. In this technique, the discharge curves obtained from a testbed were used to validate the effectiveness of the SA algorithm. Results obtained using the SA algorithm are compared to those obtained using Tremblay's parameter estimation method. The current paper uses the QPSO approach to parameterize Tremblay's model and the resultant discharge curve is compared to those generated by the GA and the PSO approaches. The simulated curves obtained from the GA, the PSO, and the QPSO parameter estimation techniques are compared to the 
experimental data together with the simulated curve obtained from Tremblay's parameter estimation method in [16], [24].

\section{PARAMETER ESTIMATION TECHNIQUES}

\section{A. Problem Formulation}

The three remarkable points identified in the battery discharge curve need to be estimated to improve the curve fitting to the experimental discharge characteristic. Additionally, the internal resistance $R$ and the maximum battery capacity $Q_{\max }$ also need to be accurately estimated since $R$ affects the shape of the battery discharge curve and $Q_{\max }$ is not provided in PHEV battery static capacity test results. In total, there are seven parameters that need to be estimated in the PHEV battery model. The objective function is defined as the RWRSS between the model discharge curve and the discharge curve obtained from the static capacity test results in [28]. Therefore, an optimization problem is formulated as follows:

$$
\begin{aligned}
\operatorname{Min}_{x} F(x) & =\operatorname{Min}_{x} \sqrt{\sum_{k=1}^{N} w_{k}\left[V_{\text {Manu }}(k)-V_{\text {Mod }}(k)\right]^{2}} \quad x \in X(2) \\
x & =\left(V_{\text {max }}, V_{\text {exp }}, Q_{\text {exp }}, V_{\text {nom }}, Q_{\text {nom }}, Q_{\text {max }}, R\right)
\end{aligned}
$$

where $F(x)$ is the objective function, $k$ is the index of the data sample, $V_{\text {mod }}(k)$ represents the model voltage at the $k^{t h}$ data sample, $V_{\text {Manu }}(k)$ is the manufacturer's voltage at the $k^{\text {th }}$ data sample, $N$ is the number of data samples, $x$ is the vector of all estimated variables, $X$ is the space of the solutions and $w_{k}$ is the weighting factor for the $k^{\text {th }}$ data sample. The manufacturer's voltage data are extracted from the static capacity test results at beginning of test (BOT) in [28], using a web-based tool called WebPlotDigitizer [29], which is used to extract data points from plots.

PHEV battery manufacturers usually limit the usable capacity of the battery to meet the battery life cycle requirements, vehicle drive performance and safety issues [30] The operational range for the state-of-charge (SOC) of PHEV batteries is between $20 \%$ and $85 \%$, i.e., the depth-of-discharge (DOD) is between $15 \%$ and $80 \%$, which is reported in [3]. In order to match the discharge curve in the operational range, an appropriate weighting function is needed. Many different mathematical expressions could be used to represent the weighting function. In this study, the weighting function used for parameter estimation is defined as follows [31]:

$$
w=\left\{\begin{array}{cr}
1 & 0.15 \leq D O D \leq 0.8 \\
\left(w_{\text {Max }}-\left(w_{\text {Max }}-w_{\text {Min }}\right) \times \frac{D O D}{0.2}\right)^{1.3} & , D O D>0.8 \\
\left(w_{\text {Min }}+\left(w_{\text {Max }}-w_{\text {Min }}\right) \times \frac{D O D}{0.2}\right)^{1.3} & , D O D<0.15
\end{array}\right.
$$

where $w_{\text {Max }}$ and $w_{\text {Min }}$ are the corresponding maximum and minimum values of the weighting function. Several other weighting functions could also be implemented to improve the accuracy of the estimated results.

\section{B. Genetic Algorithms}

GAs are population-based, stochastic search algorithms [32]. GAs have been successfully implemented in parameter identification [33] and state-of-health estimation [34]. A GA generates arbitrary initial solutions that are encoded as chromosomes that provide a correct representation of the search space [35]. The bit string representation is employed in this paper. To determine the optimal solution for the problem, the initial chromosomes are progressively modified by means of a series of actions including selection, crossover, and mutation to improve the population's fitness. The fitness of an individual chromosome is evaluated using the cost function in (2). A GA often terminates after a fixed number of iterations is reached.

\section{Particle Swarm Optimization}

The original PSO algorithm introduced by Kennedy and Eberhart [36] mimics the behavior of birds flocking and fish schooling with a swarm of particles, to simulate the search behaviors of organisms as they happen in nature [37]. The speed of movement of each particle is affected by its previous position, its personal best position $P_{\text {best }}$, and the best position of the entire swarm $G_{\text {best }}$. The positions and velocities of particles are updated as follows:

$$
\begin{gathered}
x_{i}^{k+1}=x_{i}^{k}+v_{i}^{k+1} \\
v_{i}^{k+1}=\omega v_{i}^{k}+c_{1} r_{1}\left(P_{\text {best }, i}-x_{i}^{k}\right)+c_{2} r_{2}\left(G_{\text {best }}-x_{i}^{k}\right)
\end{gathered}
$$

where $x_{i}^{k}$ is the previous position of particle $i, x_{i}^{k+1}$ is the new position of particle $i, v_{i}^{k+1}$ is the new velocity of particle $i, \omega$ is the inertia weight, $c_{1}$ and $c_{2}$ are the cognitive and social learning rates, $r_{1}, r_{2}$ are random numbers uniformly distributed between 0 and $1, P_{\text {best }, i}$ is the personal best position of particle $i$, and $G_{\text {best }}$ is the best position of the entire swarm. This paper employs a set of the PSO algorithm parameters $\omega=0.6, c_{1}=$ 1.7 and $c_{2}=1.7$ reported in [38], which is shown to exhibit fast convergenece and high robustness.

\section{Quantum-behaved Particle Swarm Optimization}

Note that the global convergence of PSO cannot be guaranteed [39] and it needs conscious efforts in parameter tuning. To further reduce the number of tuning parameters and increase the convergence rate of the PSO algorithm, a quantum-behaved particle swarm optimization (QPSO) algorithm is employed with its concept inspired by quantum mechanics and physics. The equations for updating the positions of particles are presented as follows [40]:

$$
\left\{\begin{array}{l}
x_{i}^{k+1}=P_{i}^{k}+\beta \cdot\left|m B e s t^{k}-x_{i}^{k}\right| \cdot \ln \left(\frac{1}{u}\right), k<0.5 \\
x_{i}^{k+1}=P_{i}^{k}-\beta \cdot\left|m B e s t^{k}-x_{i}^{k}\right| \cdot \ln \left(\frac{1}{u}\right), k \geq 0.5
\end{array}\right.
$$

where,

$$
\begin{gathered}
P_{i}^{k}=\left(r_{1} \cdot P_{\text {best }, i}+r_{2} \cdot G_{\text {best }}\right) /\left(r_{1}+r_{2}\right) \\
m B e s t^{k}=\frac{1}{N} \sum_{i=1}^{N} P_{\text {best }, i}
\end{gathered}
$$

where $\mathrm{mBest}^{k}$ is the mean best position defined as the mean of all the personal best positions of the swarm. $u, k, r_{1}$ and $r_{2}$ are all random numbers distributed uniformly on $(0,1)$, respectively. The one and only tuning parameter $\beta$ is the Contraction-Expansion coefficient. High values of $\beta$ are preferred in the initial stage of optimization for global exploration, whereas low values are favoured in the later stage for more accurate results in the local search [40]. This paper uses values of parameter $\beta$ that decrease dynamically from 0.9 to 0.5 during the course of iterations [41]. 


\section{E. Lower and Upper Bounds of Estimated Variables}

The admissible lower and upper bounds of each estimated variable need to be defined prior to the parameter estimation process. An inappropriate value for one estimated variable may produce irregular or abnormal discharge curves. Thus, it is crucial to reject the values generated by the parameter estimation algorithms that have spurious physical meanings. According to the PHEV battery specifications given in Table I, the lower and upper bounds of each estimated parameter can be derived using the relationships given in Table II. Some of the symbols used in Table II can be found in Fig. 1 and Table I. In Table II, the upper and lower bounds of the internal resistance are given by the maximum and minimum values of the ten-second discharge pulse resistance dataset, denoted as $R_{D O D}$, as the internal resistance is dependent on DOD variation. The ten-second discharge pulse resistance dataset shows the internal resistance with respect to the DOD and is extracted from [28] using WebPlotDigitizer. It is advised in [42] that the value of $Q_{\max }$ is $105 \%$ of the rated battery capacity. Thus, the lower and upper bounds of $Q_{\max }$ are specified as $Q_{\text {rated }}$ and $1.1 Q_{\text {rated }}$, respectively, to include additional solutions for a better estimation of $Q_{\max }$ over a wide range.

As it can be seen from Table II, the bounds of parameters $Q_{\text {exp }}$ and $Q_{\text {nom }}$ are exactly the same, this also holds true for the parameters $V_{\text {full }}, V_{\text {exp }}$, and $V_{\text {nom }}$. Special constraints should be imposed on these parameters to ensure the algorithm rejects unreasonable values being assigned into the battery model. The constraints imposed on these parameters are expressed in the following inequalities:

$V_{\text {full }}>V_{\text {exp }}>V_{\text {nom }}>V_{\text {min }}$ and $0<Q_{\text {exp }}<Q_{\text {nom }}<Q_{\text {rated }}$ (10) where $V_{\min }$ is the minimum battery pack voltage that is given as $E_{\text {min }} \cdot N_{s}$. Some of these parameters are shown schematically in Fig. 1.

\section{RESULTS \& DISCUSSION}

The PHEV battery examined in this study is the battery pack of the 2013 Chevrolet Volt - VIN 3929 [28]. The battery specifications are provided in Table I. Simulations have been conducted using 500 iterations and a population of 100 particles/chromosomes for the GA, the PSO, and the QPSO parameter estimation techniques. Table III compares the estimated Tremblay's model parameters generated by Tremblay's, GA, PSO, and QPSO parameter estimation techniques. The performances of the GA, the PSO, and the QPSO parameter estimation techniques are assessed in terms of RWRSS value, which is also presented in Table III. The performance graph of the GA, the PSO, and the QPSO parameter estimation techniques are presented in Fig. 2. The results shown in Fig. 3 compare the static capacity test curve and the simulated discharge curves estimated by Tremblay's, GA, PSO, and QPSO parameter estimation techniques.

The results shown in Fig. 3 are of significance as it can be seen that the GA, PSO, and QPSO parameter estimation techniques generate similar discharge characteristics that are almost indistinguishable in the operating SOC range for the PHEV battery. These discharge curves provide a much better model fit than the discharge characteristic estimated by
Table I. Battery specifications of the 2013 Chevrolet Volt - VIN 3929 [28].

\begin{tabular}{|c|c|}
\hline Battery Specifications & Value \\
\hline Manufacturer & LG Chem \\
\hline Rated Pack Energy/Capacity & $16.5 \mathrm{kWh} / 45.0 \mathrm{Ah}$ \\
\hline Battery Type & Lithium-Ion \\
\hline Minimum Cell Voltage $\left(E_{\min }\right)$ & $3.00 \mathrm{~V}$ \\
\hline Maximum Cell Voltage $\left(E_{\max }\right)$ & $4.15 \mathrm{~V}$ \\
\hline Nominal Cell Voltage $\left(E_{\text {nom }}\right)$ & $3.7 \mathrm{~V}$ \\
\hline Number of series-connected cells $\left(N_{s}\right)$ & 96 \\
\hline
\end{tabular}

Table II. Upper and lower bounds of each model parameter.

\begin{tabular}{|c|c|c|}
\hline Parameters & Upper Bound & Lower Bound \\
\hline $\begin{array}{c}\text { Fully Charged Voltage } \\
V_{\text {full }}(V)\end{array}$ & $1.05 E_{\max } \cdot N_{s}$ & $E_{\min } \cdot N_{s}$ \\
\hline $\begin{array}{c}\text { Voltage at the end of the } \\
\text { exponential zone } V_{\text {exp }}(V)\end{array}$ & $1.05 E_{\max } \cdot N_{s}$ & $E_{\min } \cdot N_{s}$ \\
\hline $\begin{array}{c}\text { Capacity at the end of the } \\
\text { exponential zone } Q_{\text {exp }}(A h)\end{array}$ & $Q_{\text {rated }}$ & 0 \\
\hline $\begin{array}{c}\text { Voltage at the end of the } \\
\text { nominal zone } V_{\text {nom }}(V)\end{array}$ & $1.05 E_{\text {max }} \cdot N_{s}$ & $E_{\text {min }} \cdot N_{s}$ \\
\hline $\begin{array}{c}\text { Capacity at the end of the } \\
\text { nominal zone } Q_{\text {nom }}(A h)\end{array}$ & $Q_{\text {rated }}$ & 0 \\
\hline $\begin{array}{c}\text { Maximum Capacity } \\
Q_{\text {max }}(A h)\end{array}$ & $1.1 Q_{\text {rated }}$ & $Q_{\text {rated }}$ \\
\hline Internal Resistance $R(\Omega)$ & $\mathrm{MAX}\left(R_{\text {DOD }}\right)$ & $\mathrm{MIN}\left(R_{\text {DOD }}\right)$ \\
\hline
\end{tabular}

Table III. RWRSS and model parameters obtained using the GA, the PSO, and the QPSO parameter estimation techniques.

\begin{tabular}{|c|c|c|c|c|}
\hline Model Parameter & Tremblay & GA & PSO & QPSO \\
\hline RWRSS & 172.1512 & 21.7588 & 19.1298 & 20.2075 \\
\hline $\begin{array}{c}\text { Fully Charged } \\
\text { Voltage } V_{\text {full }}(V)\end{array}$ & 398.40 & 418.32 & 404.43 & 414.88 \\
\hline $\begin{array}{c}\text { Voltage at the end } \\
\text { of the exponential } \\
\text { zone } V_{\text {exp }}(V)\end{array}$ & 376.80 & 360.36 & 332.09 & 354.33 \\
\hline $\begin{array}{c}\text { Capacity at the end } \\
\text { of the exponential } \\
\text { zone } Q_{\text {exp }}(A h)\end{array}$ & 10.67 & 22.39 & 38.42 & 27.13 \\
\hline $\begin{array}{c}\text { Nominal Voltage } \\
V_{\text {nom }}(V)\end{array}$ & 331.00 & 317.81 & 300.36 & 319.82 \\
\hline $\begin{array}{c}\text { Capacity at } \\
\text { nominal } \\
\text { voltage } Q_{\text {nom }}(A h)\end{array}$ & 42.12 & 41.70 & 43.93 & 41.17 \\
\hline $\begin{array}{c}\text { Maximum } \\
\text { Capacity } \\
Q_{\text {max }}(A h)\end{array}$ & 47.25 & 49.50 & 46.41 & 47.43 \\
\hline $\begin{array}{c}\text { Internal Resistance } \\
R(\Omega)\end{array}$ & 0.1000 & 0.1270 & 0.1340 & 0.1344 \\
\hline
\end{tabular}

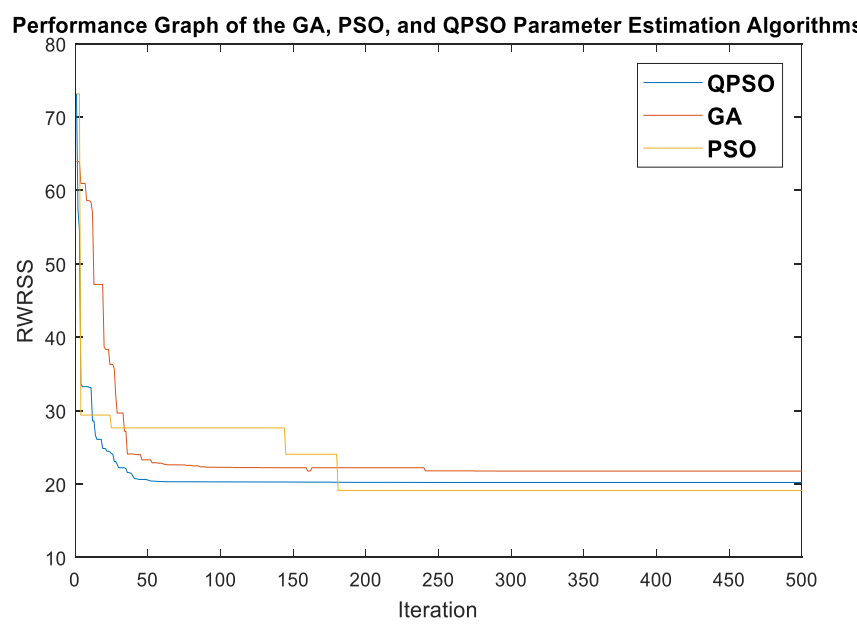

Fig. 2. The performance graph of the GA, PSO, and QPSO parameter estimation algorithms. 


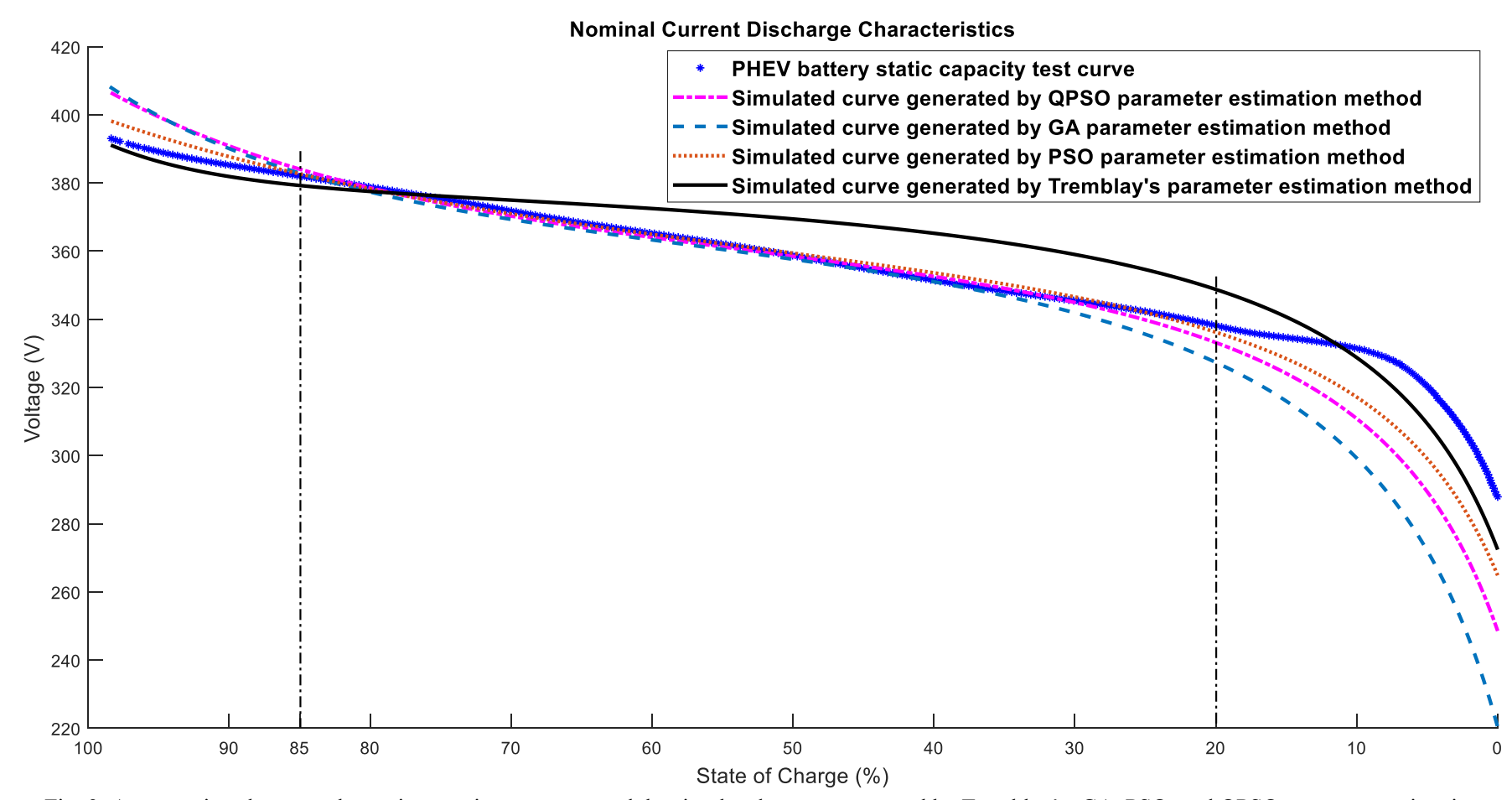

Fig. 3. A comparison between the static capacity test curve and the simulated curves generated by Tremblay's, GA, PSO, and QPSO parameter estimation techniques.

Tremblay's parameter estimation approach. From Table III, the RWRSS values for the GA, the PSO, and the QPSO are slightly different. It can be inferred that plausible global optimal solutions have been achieved by the three methods. The beginning and the end of the static capacity test curve is not well approximated by any of the four methods. This is not a major concern since these sections are beyond the scope of interest. These sections represent a difficult operating zone for vehicle propulsion since the voltage drops quite rapidly. Thus, the differences between the simulated curves and the static capacity test curve are not of importance.

From Fig. 2, it can be seen that the QPSO approach converges faster to its final RWRSS value with a similar accuracy level than the GA and the PSO approaches. The estimation process will accelerate using the QPSO algorithm and fewer iterations will be required to reach the final solution when compared to that obtained using the GA and the PSO methods. The QPSO approach also provides an advantage over the GA and the PSO algorithms that there is only one tuning parameter $\beta$ involved in the QPSO algorithm, as shown in (7), thus the tuning effort is significantly reduced when compared to that of the GA and the PSO approaches.

\section{CONCLUSIONS \& FUTURE WORK}

This paper uses the QPSO parameter estimation technique to estimate Tremblay's battery model parameters. The parameterization of Tremblay's battery model has been formulated as a multivariable optimization problem that can be solved using the QPSO parameter estimation technique. The simulated discharge curve generated by the QPSO approach is compared with those obtained using the GA and PSO approaches. The QPSO parameter estimation technique generates solutions with similar accuracy levels when compared to those obtained using the GA and the PSO parameter estimation techniques, and converges to the final solution with fewer iterations than the other methods. This highlights the potential enhancement of using the QPSO algorithm to parameterize Tremblay's model as the QPSO algorithm only requires one parameter $\beta$ to be tuned.

Future work may include additional parameter estimation techniques, such as the hybrid optimization technique mentioned in [8], to improve the performance of Tremblay's model parameterization. The performances of the GA, the PSO, and the QPSO parameter estimation techniques could be further enhanced by optimizing the algorithms' parameters, such as the cognitive and social learning rates in the PSO algorithm and the contraction-expansion coefficient in the QPSO algorithm. The weighting function could also be adjusted to improve the convergence speed and accuracy of the estimated results. Finally, additional simulations could be performed using a larger particle/chromosome size and a higher number of generations.

\section{REFERENCES}

[1] A. Nagarajan and W. Shireen, "Grid connected residential photovoltaic energy systems with Plug-In Hybrid electric Vehicles (PHEV) as energy storage," Power Energy Soc. Gen. Meet., pp. 1-5, 2010.

[2] F. R. Islam, H. R. Pota, M. S. Rahman, and M. S. Ali, "Performance analysis of photovoltaic cell with dynamic PHEV loads," in Univ. Power Eng. Conf. (AUPEC), 2012 22nd Australas., 2012, pp. 1-6.

[3] S. Shao, M. Pipattanasomporn, and S. Rahman, "Grid Integration of Electric Vehicles and Demand Response With Customer Choice," IEEE Trans. Smart Grid, vol. 3, no. 1, pp. 543-550, Mar. 2012.

[4] L. Jian, H. Xue, G. Xu, X. Zhu, D. Zhao, and Z. Y. Shao, "Regulated charging of plug-in hybrid electric vehicles for minimizing load variance in household smart microgrid," IEEE Trans. Ind. Electron., vol. 60, no. 
8, pp. 3218-3226, 2013.

[5] K. Clement-Nyns, E. Haesen, and J. Driesen, "The impact of charging plug-in hybrid electric vehicles on a residential distribution grid," IEEE Trans. Power Syst., vol. 25, no. 1, pp. 371-380, Feb. 2010.

[6] M. R. Khalghani, S. Khushalani-Solanki, and J. Solanki, "Optimal integration and location of PHEV aggregators in power distribution systems," in 2016 North Am. Power Symp., 2016, pp. 1-6.

[7] F. Altaf and B. Egardt, "Comparative Analysis of Unipolar and Bipolar Control of Modular Battery for Thermal and State-of-Charge Balancing," IEEE Trans. Veh. Technol., vol. 66, no. 4, pp. 2927-2941, Apr. 2017.

[8] D. Gallo, C. Landi, M. Luiso, and R. Morello, "Optimization of Experimental Model Parameter Identification for Energy Storage Systems," Energies, vol. 6, no. 9, pp. 4572-4590, Sep. 2013.

[9] Xuewei Qi, G. Wu, K. Boriboonsomsin, and M. J. Barth, "An on-line energy management strategy for plug-in hybrid electric vehicles using an Estimation Distribution Algorithm," in 17th International IEEE Conference on Intelligent Transportation Systems (ITSC), 2014, pp. 2480-2485.

[10] N. Omar et al., "Rechargeable Energy Storage Systems for Plug-in Hybrid Electric Vehicles-Assessment of Electrical Characteristics," Energies, vol. 5, no. 12, pp. 2952-2988, Aug. 2012.

[11] S. Yuan, H. Wu, and C. Yin, "State of charge estimation using the extended Kalman filter for battery management systems based on the ARX battery model," Energies, vol. 6, no. 1, pp. 444-470, 2013.

[12] D. Aurbach, "Characterization of Batteries by Electrochemical and nonElectrochemical Techniques," in Ind. Appl. Batter. From Cars to Aerosp. Energy Storage, 2007, pp. 119-131.

[13] H. He, R. Xiong, and J. Fan, "Evaluation of lithium-ion battery equivalent circuit models for state of charge estimation by an experimental approach," Energies, vol. 4, no. 4, pp. 582-598, 2011.

[14] G. Liu, M. Ouyang, L. Lu, J. Li, and X. Han, "Online estimation of lithium-ion battery remaining discharge capacity through differential voltage analysis," J. Power Sources, vol. 274, pp. 971-989, Jan. 2015.

[15] Y. Wang and L. Li, "Li-ion battery dynamics model parameter estimation using datasheets and particle swarm optimization," Int. J. Energy Res., vol. 40, no. 8, pp. 1050-1061, Jun. 2016.

[16] O. Tremblay and L.-A. A. Dessaint, "Experimental validation of a battery dynamic model for EV applications," World Electr. Veh. J., vol. 3, no. 1, pp. 289-298, 2009.

[17] H. Oman, "On-board energy and power management on electric vehicles: effect of battery type," in 17th DASC. AIAA/IEEE/SAE. Digital Avionics Systems Conference. Proceedings (Cat. No.98CH36267), 1998, vol. 2, p. I43/1-I43/6.

[18] J. Gonder and T. Markel, "Energy management strategies for plug-in hybrid electric vehicles," in 2007 SAE World Congress, 2007, pp. 1-5.

[19] M. Alhanouti, M. Gießler, T. Blank, and F. Gauterin, "New ElectroThermal Battery Pack Model of an Electric Vehicle," Energies, vol. 9, no. 7 , p. 563, 2016.

[20] H. He, R. Xiong, H. Guo, and S. Li, "Comparison study on the battery models used for the energy management of batteries in electric vehicles," Energy Convers. Manag., vol. 64, pp. 113-121, Dec. 2012.

[21] G. Liu et al., "A comparative study of equivalent circuit models and enhanced equivalent circuit models of lithium-ion batteries with different model structures," IEEE Transp. Electrif. Conf. Expo, ITEC Asia-Pacific 2014 - Conf. Proc., pp. 1-6, 2014.

[22] X. Hu, S. Li, and H. Peng, "A comparative study of equivalent circuit models for Li-ion batteries," J. Power Sources, vol. 198, pp. 359-367, Jan. 2012.

[23] B. Schweighofer, K. M. Raab, and G. Brasseur, "Modeling of high power automotive batteries by the use of an automated test system," IEEE Trans. Instrum. Meas., vol. 52, no. 4, pp. 1087-1091, 2003.

[24] O. Tremblay, L.-A. Dessaint, and A.-I. Dekkiche, "A Generic Battery Model for the Dynamic Simulation of Hybrid Electric Vehicles," in IEEE Veh. Power Propuls. Conf., 2007, pp. 284-289.

[25] J. P. Christopherson, "Battery Test Manual For Electric Vehicles Revision 3," Idaho Falls, USA, 2015.

[26] R. Xiong, H. He, H. Guo, and Y. Ding, "Modeling for lithium-ion battery used in electric vehicles," Procedia Eng., vol. 15, pp. 2869-2874, 2011.

[27] M. D. F. Brondani, A. T. Z. R. Sausen, P. S. Sausen, and M. O. Binelo, "Battery Model Parameters Estimation Using Simulated Annealing," TEMA (São Carlos), vol. 18, no. 1, p. 127, May 2017.
[28] Chevrolet Volt - VIN 3929 (2013), "Advanced Vehicle Testing Activity - Battery Pack Laboratory Testing Results,” U.S. Dept. of Energy, 2015. [Online].

Available: https://avt.inl.gov/sites/default/files/pdf/phev/batteryVolt3929.pdf.

[29] A. Rohatgi, "WebPlotDigitizer," 2016. [Online]. Available: $\mathrm{http}: / /$ arohatgi.info/WebPlotDigitizer.

[30] A. G. Boulanger, A. C. Chu, S. Maxx, and D. L. Waltz, "Vehicle electrification: Status and issues," Proc. IEEE, vol. 99, no. 6, pp. 1116$1138,2011$.

[31] M. Negnevitsky, "Artificial Intelligence: A Guide to Intelligent Systems," 3rd ed., MA: Addison-Wesley, 2011, pp. 224-225.

[32] S. Katare, A. Bhan, J. M. Caruthers, W. N. Delgass, and V. Venkatasubramanian, "A hybrid genetic algorithm for efficient parameter estimation of large kinetic models," Comput. Chem. Eng., vol. 28, no. 12, pp. 2569-2581, 2004.

[33] L. Zhang, L. Wang, G. Hinds, C. Lyu, J. Zheng, and J. Li, "Multiobjective optimization of lithium-ion battery model using genetic algorithm approach," J. Power Sources, vol. 270, pp. 367-378, Dec. 2014.

[34] Z. Chen, C. C. Mi, Y. Fu, J. Xu, and X. Gong, "Online battery state of health estimation based on Genetic Algorithm for electric and hybrid vehicle applications," J. Power Sources, vol. 240, pp. 184-192, Oct. 2013.

[35] Y. Zhang, S. Lyden, B. A. León de la Barra, and M. E. Haque, "A genetic algorithm approach to parameter estimation for PV modules," in 2016 IEEE Power and Energy Society General Meeting (PESGM), 2016, pp. $1-5$.

[36] J. Kennedy and R. Eberhart, "Particle swarm optimization," Neural Networks, 1995. Proceedings., IEEE Int. Conf., vol. 4, pp. 1942--1948 vol.4, 1995.

[37] J. Liu, "Evolving Particle Swarm Optimization Implemented by a Genetic Algorithm," J. Adv. Comput. Intell. Intell. Informatics, vol. 12, no. 3, pp. 284-289, May 2008.

[38] J. L. Kuo, K. L. Chao, and L. S. Lee, "Dual mechatronic MPPT controllers with PN and OPSO control algorithms for the rotatable solar panel in PHEV system," IEEE Trans. Ind. Electron., vol. 57, no. 2, pp. 678-689, 2010.

[39] F. Van Den Bergh, "An analysis of particle swarm optimizers," University of Pretoria, Pretoria, Gauteng, South Africa, 2001.

[40] S. N. N. Omkar, R. Khandelwal, T. V. S. V. S. Ananth, G. Narayana Naik, and S. Gopalakrishnan, "Quantum behaved Particle Swarm Optimization (QPSO) for multi-objective design optimization of composite structures," Expert Syst. Appl., vol. 36, no. 8, pp. 11312$11322,2009$.

[41] D. Su, W. Xu, and J. Sun, "Quantum-behaved particle swarm optimization with crossover operator," Int. Conf. Wirel. Networks Inf. Syst. WNIS 2009, pp. 399-402, 2009.

[42] MathWorks, "Implement generic battery model - Simulink," The MathWorks, Inc., 2017. [Online]. Available: https://au.mathworks.com/help/physmod/sps/powersys/ref/battery.html. [Accessed: 27-Jun-2016]. 